\title{
Special issue on Hybridization of Intelligent Systems
}

\begin{abstract}
Hybridization of intelligent systems is a promising research field of computational intelligence focusing on synergistic combinations of multiple approaches to develop the next generation of intelligent systems. A fundamental stimulus to the investigations of Hybrid Intelligent Systems (HIS) is the awareness that combined approaches will be necessary to solve challenging problems in complex real-world situations. Neural computing, machine learning, fuzzy logic, evolutionary algorithms, agent-based methods, swarm optimization, quantum computing, are some intelligent systems - among others - that have been established
\end{abstract} and have shown their strengths and drawbacks.

Giving the attribution of being "intelligent" to a system seems to be only admissible, if the system itself is sufficiently complex. However, all attempts to pose a structure on such complex systems show that the chosen structural components become intermingled, as the systems' modeling itself tangents a practical application. If there is a layer model, the layers start to interact; if there are components, they cease to act independently from each other; and if there are rules, the rule conclusions will start to depend on context that is not captured by the rule antecedents.

Compared to other systems' features like dimension or scale, intelligence seems to be a vague system feature - it does not appear to be a property of a system that can be easily put into mathematical terms and equations, and whose presence can be easily verified by appropriately designed tests or experiments. Thus, intelligence is a cross-layer, cross-component and cross-rule specific matter, i.e. hybrid in its essence.

In this special issue of the International Journal of Hybrid Intelligent Systems (IJHIS), we are presenting four excellent contributions, which demonstrate how intelligent systems emerge from hybridization. The focus is always on a complex system, be it the evolution- ary design of image processing operators, the evolutionary reinforcement learning of neural networks, or the development of new general universally applicable learning strategies based on ensemble classification or clustering.

The contributions are based on papers that were presented at the "Sixth International Conference on Hybrid Intelligent Systems", which was held in conjunction with the "Fourth Conference on Neuro-Computing and Evolving Intelligence" (HIS-NCEI 2006) from December 13 to 15, 2006, at the Auckland University Technology Park in Auckland, New Zealand. About 70 papers devoted to the field of hybrid intelligent systems were presented at this conference.

In the paper "Multi-objective Clustering Ensemble" by K. Faceli and M.C.P. de Souto, authors expand on recent works in multi-objective clustering and ensemble clustering. In the presented evolutionary approach, different individuals represent different clustering results, according to the use of several clustering methods with different parameter settings. New clustering results are produced from combination of existing ones in each generation, and multiple objectives are used for selecting among the various partitions. The proposed system is applied to synthetic data for verification, and to UCI benchmark data for proving efficiency.

Similar to clustering ensembles, an increasing number of recent studies also considered the ensemble approach for classification. Particular attention was given to methods that combine a larger number of simple classifiers. Usually, these classifiers are specified in advance. In the paper "Genetic Rule Selection with a Multi-Classifier Coding Scheme for Ensemble Classifier Design" by Y. Nojima and H. Ishibuchi, authors are going a different way by actually generating the ensemble from a larger number of simple (interval-based) classifiers. The majority-voting results of the gener- 
ated ensemble classifier give a means for applying an evolutionary algorithm, and the authors present a corresponding encoding. Following a general insight into ensemble classifiers, which is, roughly speaking, that they only make sense if the classifier results are divergent, authors also study the use of an entropy-based measure for classifier diversity as an additional objective, and apply multi-objective optimization. Tested on a number of UCI problems, the use of that second measure could improve the final results in most of the cases.

Hybrid approaches also appeared to be useful for the design of the classifiers itself. From an evolutionary perspective, each individual can represent a single classifier, with the classifier performance being the single objective that should be maximized. However, a number of problems, especially incomparability of rates of differently structured classifiers and high computational effort, make this approach hard to handle in practice. The paper "Evolutionary Reinforcement Learning of Artificial Neural Networks" by Nils T. Siebel and Gerald Sommer shows a different way by gradually developing the neural network structure, starting from a simple structure and introducing sub-networks and distant links between neurons, as specified in a corresponding genetic encoding. At the same time that a neural net- work structure is expanded, its parameters are adjusted as well, to gain optimal performance of the modified network. The approach is demonstrated on a visual servoing task.

Last but not least, in the paper "Optimized Texture Operators for the Automated Design of Image Analysis Systems - Non-linear and oriented kernels vs. gray value co-occurrence matrices," the application is the evolutionary adaptation of an image processing system. Here, evolutionary computation and particle swarm optimization are compared for the generation of nonlinear kernel based image processing filters.

We hope that this selection of high-ranked papers from the HIS-NCEI 2006 conference gives a contribution to emerging issues in the interplay of different soft computing techniques for the design of hybrid intelligent systems.

\section{Guest Editors}

Mario Köppen

Kyushu Institute of Technology E-mail: mkoeppen@pluto.ai.kyutech.ac.jp

Richard Weber University of Chile E-mail: rweber@dii.uchile.cl 